\title{
Morphology Transition and Layer-by-Layer Growth of $\mathrm{Rh}(111)$
}

\author{
F. Tsui,* J. Wellman, C. Uher, and Roy Clarke \\ Department of Physics, University of Michigan, Ann Arbor, Michigan 48109
}

(Received 2 August 1995)

\begin{abstract}
We have observed a morphological transition in the nucleation and growth of epitaxial $\mathrm{Rh}(111)$. The transition occurs near $600 \mathrm{~K}$ and is signaled by a change in the shape of the surface features from fingered to compact. The transition appears to be related to a change in the critical nucleation size. On both sides of the transition, there lies a regime of persistent layer-by-layer growth. The general surface features exhibit well-defined length scales and as growth proceeds they increase in size following a power-law dependence on film thickness with a morphology-independent exponent of $0.33 \pm 0.03$. The results suggest a general pathway to the layer-by-layer growth of close-packed metals. [S0031-9007(96)00058-0]
\end{abstract}

PACS numbers: 68.55.Jk, 61.16.Ch, 68.35.Bs

Recent epitaxial growth experiments [1-4] show the presence of surface features that exhibit characteristic length scales. The observed three-dimensional (3D) features arise from instabilities due to the competition between various diffusion barriers near step edges [5]. These findings suggest that the non-self-affine morphologies may be a common occurrence when surface diffusion is sufficiently large with profound implications for the growth of thin films in this regime. In particular, several models [6,7] have focused on the power-law evolution of surface feature size with respect to the average film thickness, leading to predictions which our experimental results can address.

Quantitative experimental data on the thickness dependence are still very sketchy and many important questions are yet to be addressed: How is the evolution of these 3D features related to layer-by-layer growth, what are the effects of "kinks" at step edges, and what role does initial nucleation play? In this Letter we report on the evolution of surface morphology during epitaxial growth of a model system, $\mathrm{Rh}(111)$, as a function of temperature, thickness, and growth rate. We show that surface features including those resulting from layer-by-layer growth generally exhibit characteristic dimensions which scale with thickness and temperature, and that a global transformation of the surface morphology accompanied by layer-by-layer growth arises from a change in the critical nucleation size. The results have important general implications for the growth of close-packed metal films.

Our studies were performed in a VG $80 \mathrm{M}$ molecular beam epitaxy (MBE) chamber equipped with an in situ scanning tunneling electron microscope (STM) and a real-time reflection high energy electron diffraction (RTRHEED) imaging system [8]. The growth template for the study consists of a synthetic fluorine mica substrate [9] and a buffer layer of 250 monolayers (ML) Rh. We chose this system for two main reasons. First, as a classic close-packed system, $\mathrm{Rh}$ is much less susceptible to contamination and chemical reaction than most metals; and second, it is known [9] that $\mathrm{Rh}$ grows well on the fcc (111) face on synthetic mica.

The mica substrates were freshly cleaved before introduction into the MBE chamber, and then degassed at $1200 \mathrm{~K}$. The $\mathrm{Rh}$ buffer was subsequently grown at $600 \mathrm{~K}$ and then annealed at $1100 \mathrm{~K}$, giving typical terrace lengths of several thousand $\AA$. The $\mathrm{Rh}$ was evaporated from an electron-beam hearth with respective rates of 1 and $2 \mathrm{ML} / \mathrm{min}$ for the nucleation and the growth studies. The base pressure of the MBE chamber was maintained at below $10^{-10}$ torr. The samples were cooled at $50 \mathrm{~K} / \mathrm{min}$ after growth was terminated, and STM images, the frozenin configurations, were then taken at room temperature in constant current mode with a typical current of $1 \mathrm{nA}$ and a bias of $-0.1 \mathrm{~V}$. Continuous RHEED observations confirm that there is no change in the surface morphology during cooling.

The strong influence of growth temperature on surface morphology and $\mathrm{Rh}(111)$ is demonstrated by the STM images of a series of 10.1 ML films grown at different temperatures, as shown in Fig. 1. Several important features are immediately apparent: (1) the surface features show a characteristic length scale typified by islands with well-defined size and separation; (2) the shape of the features exhibits a transition near $600 \mathrm{~K}$ from compact at high growth temperatures [Figs. 1(d)-1(f)] where the step edges are smooth, to fingered at low temperatures [Figs. 1(a)-1(c)] where the step edges have "kinks"; and (3) the height of the features has a minimum near the transition, where we also observe layer-by-layer growth. The presence of a layer-by-layer growth regime between 500 and $700 \mathrm{~K}$ is supported by persistent RHEED oscillations. These observations reinforce the view that surface features are generally not self-affine when diffusion is sufficiently high [1] and that the morphology of layer-by-layer growth is a special case simply because the coherent nature of the process predicates well defined length scales.

Quantitative analysis of the STM images reveals that the surfaces exhibit sharp ringlike Fourier components 


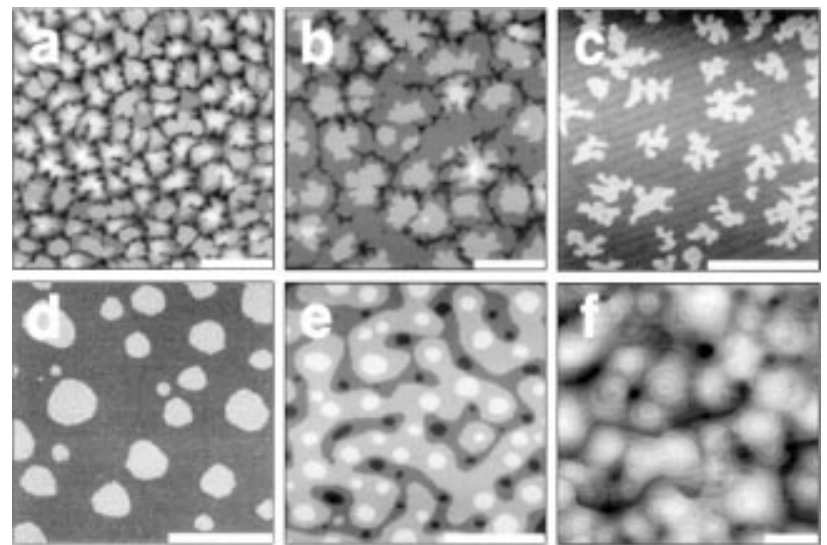

FIG. 1. STM images of 10.1 ML Rh(111) surfaces grown at various temperatures, (a) $375 \mathrm{~K}$, (b) $450 \mathrm{~K}$, (c) $530 \mathrm{~K}$, (d) $680 \mathrm{~K}$, (e) $725 \mathrm{~K}$, and (f) $810 \mathrm{~K}$. The horizontal white bars correspond to $400 \AA$ for images (a)-(e), and $1000 \AA$ for (f).

in the growth plane and long-ranged oscillations in the height-height correlation functions as well, thus confirming the presence of non-self-affine features. The temperature dependences of the average height $W$ and separation $R$ of the surface features are shown in Fig. 2. The broad minimum in mean feature height $W$ [Fig. 2(a)] is accompanied by a break in the otherwise monotonically decreasing aspect ratio $W / R$ as temperature increases [Fig. 2(b)], a very distinct signature of the morphological transition. The general decreasing trend of $W / R$ indicates the important role played by capillary-induced smoothing due to diffusion [5], but the fact that the surface area does not decrease monotonically with increasing temperature and that mounds do form both indicate the importance of asymmetric diffusion barriers at step edges [5]. The break of $W / R$, on the other hand, is completely consistent with capillary effects alone at a morphological transition from fingered to compact.

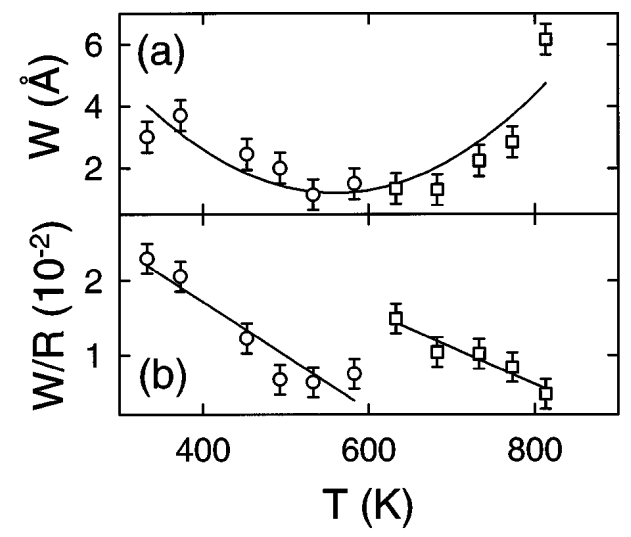

FIG. 2. Size and shape of the surface features on 10.1 ML $\mathrm{Rh}(111)$ as a function of temperature. (a) Feature height $W$, the rms height from average film thickness in $\AA$, and (b) feature aspect ratio $W / R$, average height $W$ versus average separation $R$. Points above and below the morphological transition near $600 \mathrm{~K}$ are indicated by squares and circles, respectively. Lines are to guide the eye.
One of the key issues in the formation of surface features is the nature of diffusion at step edges. As seen in Fig. 1, the compact mounds formed above the morphological transition exhibit some reflection symmetry (mounds and craters) not shown by the low temperature fingered features. The asymmetry at low temperature is thought to be associated with the kinetics of step propagation for terraces at the top and at the bottom of the mounds [4], and, moreover, the morphology transition observed here suggests that the shape of the step edges plays an important role in the kinetics.

It is interesting to point out that the observed layerby-layer growth occurs on both sides of the morphology transition. This indicates that the presence of kinks at the step edges [10,11] and island spacing [12] are two important factors in producing smooth growth. Just below the transition the presence of kinks modifies the diffusion barriers by introducing wells and barriers along the step edges [10], thus facilitating the layerby-layer growth. In this regime our experiments show that increasing the growth rate will increase the chance for nucleation and induce rougher growth. Above the transition the compact mounds become smaller and closer as temperature decreases [Fig. 2(b)], and when they reach a critical size [12] (in this case $\sim 100 \AA$ A separation) layerby-layer growth occurs. In this regime, on the other hand, our experiments reveal that increasing the growth rate reduces island spacing and promotes the likelihood of producing layer-by-layer growth at higher temperatures. However, the origin of the observed large critical size is unclear.

What ultimately triggers the transition? The strong dependence of diffusion barriers on the local geometries, particularly those along as well as across the step edges, suggests that the answer lies in the nucleation process $[11,13]$. We have carried out a detailed investigation of the initial nucleation at the submonolayer level, and the results reveal that the critical cluster size also exhibits a transition near $600 \mathrm{~K}$. Figure 3 shows the island density as a function of temperature and island size distribution for a Rh submonolayer coverage of $0.1 \mathrm{ML}$. The change of slope in the island density $N$ shown in Fig. 3(a) indicates a change in the activation energy [14,15], separating the diffusion process into two branches. In addition, the island size distribution exhibits two different scaling functions [15-19], as shown in Figs. 3(b) and $3(\mathrm{c})$. In the low coverage limit with isotropic diffusion, the island density $N$ is given by [20]

$$
N \sim \exp \left[\left(i E_{d}+E_{i}\right) /(i+2) k_{B} T\right],
$$

where $E_{d}$ is the activation energy for diffusion, and $E_{i}$ is the binding energy for the critical cluster size $i$, above which clusters form stable islands; e.g., $i=1$ corresponds to stable dimers, etc. Models based on critical cluster size predict that for each $i$ there is a unique distribution function [16-19]. Scaling functions shown in Figs. 3(b) 


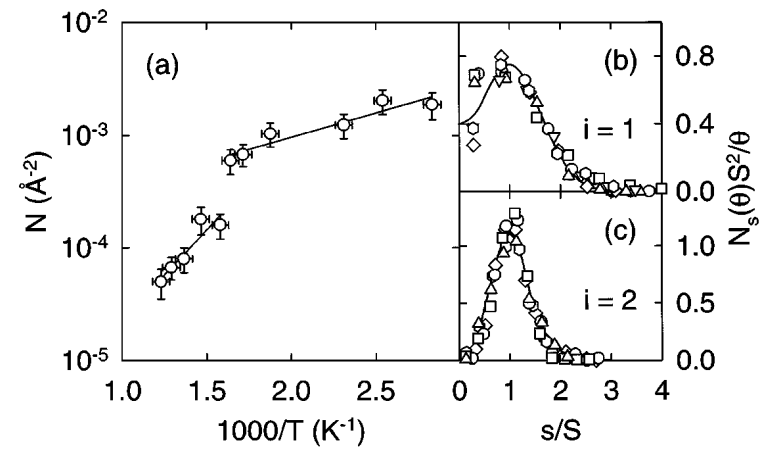

FIG. 3. (a) Arrhenius plot of island density, $N$ in $\AA^{-2}$, for Rh coverage $\theta$ of $0.1 \mathrm{ML}$ as a function of inverse temperature. The slopes indicate respective activation energies of $0.06 \pm$ $0.02 \mathrm{eV}$ at the low temperature branch (right side of the graph) and $0.25 \pm 0.08 \mathrm{eV}$ at high temperatures (left side of the plot). Scaling of island size distributions for temperatures (b) below $600 \mathrm{~K}$ and (c) above $600 \mathrm{~K}$. Temperatures are (b) $600 \mathrm{~K}$ (circles), $580 \mathrm{~K}$ (up triangles), $530 \mathrm{~K}$ (down triangles), $430 \mathrm{~K}$ (squares), $390 \mathrm{~K}$ (hexagons), and $350 \mathrm{~K}$ (diamonds); (c) $770 \mathrm{~K}$ (up triangles), $730 \mathrm{~K}$ (diamonds), $700 \mathrm{~K}$ (circles), $680 \mathrm{~K}$ (squares), and $630 \mathrm{~K}$ (hexagons). Number of islands per area at size $s$ and coverage $\theta, N_{s}(\theta)$, is normalized by $S^{2} / \theta$ with $S$ the average island size, and $s$ is scaled by $S$. Lines in (b) and (c) are to guide the eye.

and 3(c) yield the critical cluster size of $i=1$ below $600 \mathrm{~K}$ and $i=2$ above [16-19]. From Eq. (1) this leads to $E_{d}=0.18 \pm 0.06 \mathrm{eV}$ and $E_{2}=0.6 \pm 0.4 \mathrm{eV}$. Note that these are average activation energies for a nearly isotropic (111) surface. The measured "one-bond" energy is comparable to the reported experimental estimate of $0.5 \pm 0.5 \mathrm{eV}$ for $\mathrm{Fe}(100)$ [15] as well as the Monte Carlo and rate equation estimates of $0.6 \pm 0.1$ and $0.7 \mathrm{eV}$, respectively $[16,18]$. The measured $E_{d}$ also is comparable to a previously published value for $\mathrm{Rh}(111)$ of $0.15 \pm$ $0.02 \mathrm{eV}$ [21].

While the diffusion along step edges is clearly a crucial factor in determining the final morphology of the system [13], it is the initial "seed" clusters that set up the edge-diffusion process. In the stable dimer regime ( $i=1$ case), the presence of thickened fingers [Fig. 1(c)] indicates some edge diffusion. However, in the regime of stable trimers ( $i=2$ case) additional processes become available, including one-bond detachment (unstable dimers) which can trigger more edge diffusion. This can lead to the formation of compact islands in the $i=2$ regime. A similar transition is predicted for a square lattice even without edge diffusion [16,19]. Therefore the observed change in critical cluster size is evidently large enough to give rise to the observed global morphological transition accompanied by layer-by-layer growth. We have also observed a similar transition during the annealing of films deposited at temperatures below $600 \mathrm{~K}[22]$.

Finally we examine the evolution of mounds as a function of film thickness at temperatures above and below the morphological transition. Figure 4 illustrates the process at $725 \mathrm{~K}$. The initial growth is layer-by-layer accompanied by RHEED oscillations. After the initial several monolayers the aspect ratio of the surface features rapidly increases, within a couple of monolayers, to a stable value (the so-called "magic slope" [1-4]) [Fig. 4(d)]. The separation, however, evolves smoothly and continuously from the initial nucleation at the submonolayer level, as shown in Fig. 4(e), similar in appearance to those reported in $\mathrm{Fe}(001)$ [4]. As growth proceeds, the surface features maintain their shape but increase in size, so they both scale and coarsen, as demonstrated in Figs. 4(a)4(c). The coarsening process follows a power-law dependence on film thickness $t$ with an exponent of $0.33 \pm 0.03$ [Fig. 4(e)]. This exponent is not predicted by existing models, and it may have implications for the underlying physics of the asymmetric diffusion barrier. For growth temperatures above and below the morphological transition the coarsening exhibits the same exponent. The fact that the same exponent is obtained for growth within regimes where the strength and spatial extent of the asymmetric step-edge barriers are different indicates that the exponent is independent of the size and shape of the barrier.

A splitting of RHEED streaks occurs for growth below $350 \mathrm{~K}$, which corresponds to the characteristic separation of the mounds $[4,14]$. This gives a second independent measure of the separation in addition to STM images.
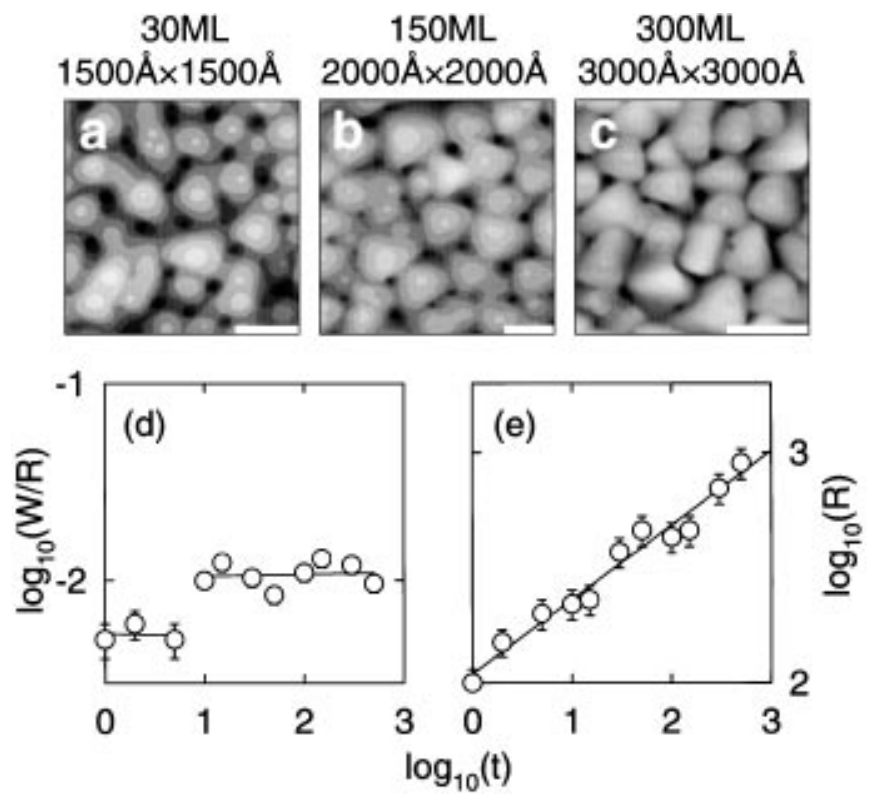

FIG. 4. Coarsening of surface features for growth at $725 \mathrm{~K}$. (a)-(c) STM images of 30, 150, and $300 \mathrm{ML}$ Rh surfaces, respectively. The horizontal white bars correspond to $400 \AA$ for images (a) and (b), and $1000 \AA$ for (c). (d) Log-log plot of the aspect ratio of surface features, $W / R$, and (e) log-log plot of the feature separation $R$ in $\AA$ as a function of film thickness $t$ in ML. The line in (e) indicates a power law with an exponent of $0.33 \pm 0.03$. 
It is interesting to point out that the rounded triangular shape of the mounds shown in Figs. 4(a)-4(c) reflects the threefold fcc symmetry, indicating the absence of stacking faults; in fact, we do see hexagonal features when impurities are present.

In summary, we have studied the nucleation and growth of epitaxial Rh(111) using STM and RT-RHEED. The $\mathrm{Rh}$ surface exhibits a morphological transition near $600 \mathrm{~K}$, about $1 / 4$ of the $\mathrm{Rh}$ melting temperature, where surface features change from fingered to compact. This is accompanied by a minimum in surface roughness and by persistent layer-by-layer growth. Detailed studies of the nucleation statistics indicate that a transition in critical cluster size from one to two atoms can trigger a morphological transition by influencing local geometry. A second characteristic feature is the presence of mounds that exhibit well defined shapes and sizes. Both compact and fingered mounds maintain their shape but increase in size as growth proceeds following a power-law dependence on film thickness with a universal exponent of $1 / 3$. While the presence of kinks along the step edges modifies the diffusion barrier, it does not alter the nature of the coarsening process, and the observed exponent seems to be independent of the size and shape of the step-edge barrier. Our results reveal that the occurrence of a global morphological transition during MBE growth can lead to layer-by-layer growth on both sides of the transition, a special regime in the evolution of non-self-affine surface morphologies. Our findings for epitaxial $\mathrm{Rh}$ should be generally applicable to other epitaxial systems, particularly metallic (111) surfaces. In a wider context, the appearance of global morphological transitions triggered by local symmetry breaking raises several interesting questions for atomistic growth models.

We are grateful to C.P. Flynn for discussions and for providing synthetic mica substrates. We thank L. M. Sander, B.G. Orr, A. Zangwill, and J.G. Amar for stimulating discussions, and C. Taylor, D. Barlett, and D. Litvinov for assistance. The work was supported in part by ONR Grant No. N00014-92-J-1335.

*Permanent address: Department of Physics and Astronomy, University of North Carolina, Chapel Hill, NC 27599-3255.
[1] M. D. Johnson, C. Orme, A. W. Hunt, D. Graff, J. L. Sudijono, L. M. Sander, and B. G. Orr, Phys. Rev. Lett. 72, 116 (1994); C. Orme, M. D. Johnson, J. L. Sudijono, K. T. Leung, and B. G. Orr, Appl. Phys. Lett. 64, 860 (1994).

[2] H. J. Ernst, E. Fabre, R. Folkerts, and J. L. Lapujoulade, Phys. Rev. Lett. 72, 112 (1994); J. Vac. Sci. Technol. 12, 1 (1994).

[3] J. van Nostrand, S. J. Chey, M. A. Hasan, D. G. Cahill, and J. E. Greene, Phys. Rev. Lett. 74, 1127 (1995).

[4] J. A. Stroscio, D. T. Pierce, M. Stiles, A. Zangwill, and L. M. Sander, Phys. Rev. Lett. 75, 4246 (1995).

[5] J. Villain, J. Phys. I (France) 1, 19 (1991).

[6] A. W. Hunt, C. Orme, D. R. M. Williams, B. G. Orr, and L. M. Sander, Europhys. Lett. 27, 611 (1994); in Scale Invariance, Interfaces and Non-Equilibrium Dynamics, edited by A. J. McKane (Plenum, New York, 1994).

[7] M. Siegert and M. Plishchke, Phys. Rev. Lett. 73, 1517 (1994); M. Siegert, in Scale Invariance, Interfaces and Non-Equilibrium Dynamics (Ref. [6]).

[8] D. Barlett, C. W. Snyder, B. G. Orr, and R. Clarke, Rev. Sci. Instrum. 62, 1263 (1991); data acquisition using KSA300, k-Space Assoc. Inc., Ann Arbor, MI 48104.

[9] F. Tsui, P.D. Han, and C. P. Flynn, Phys. Rev. B 47, 13648 (1993).

[10] J. Jacobsen, K. Jacobsen, P. Stoltze, and J. K. Nørskov, Phys. Rev. Lett. 74, 2295 (1995).

[11] R. Kunkel, B. Poelsema, L. L. Verheij, and G. Comsa, Phys. Rev. Lett. 65, 733 (1990).

[12] J. Tersoff, A. W.D. van der Gon, and R. M. Tromp, Phys. Rev. Lett. 72, 266 (1994).

[13] H. Röder, K. Bromann, H. Brune, and K. Kern, Phys. Rev. Lett. 74, 3217 (1995).

[14] J. A. Stroscio, D. T. Pierce, and R. A. Dragoset, Phys. Rev. Lett. 70, 3615 (1993).

[15] J. A. Stroscio and D. T. Pierce, Phys. Rev. B 49, 8522 (1994).

[16] J. G. Amar and F. Family, Phys. Rev. Lett. 74, 2066 (1995); (to be published).

[17] G. S. Bales and D.C. Chrzan, Phys. Rev. B 50, 6057 (1994).

[18] M. C. Bartelt and J.W. Evans, Phys. Rev. B 46, 12675 (1992); Surf. Sci. 298, 421 (1993); J. W. Evans and M. C. Bartelt, J. Vac. Sci. Technol. A 12, 1800 (1994).

[19] C. Ratsch, A. Zangwill, P. Šmilauer, and Vvedensky, Phys. Rev. Lett. 72, 3194 (1994).

[20] J. A. Venables, Philos. Mag. 27, 697 (1973).

[21] G. Ayrault and G. Ehrlich, J. Chem. Phys. 60, 281 (1974).

[22] F. Tsui, J. Wellman, C. Uher, and R. Clarke (unpublished). 